\title{
Role of Modern Sonography in the Assessment of Knee Pathologies: Review of a Large Series of Patients
}

\author{
Saher Srour ${ }^{1,2 *}$ and Rabih Fares ${ }^{2}$ \\ ${ }^{1}$ MRI unit, Department of diagnostic radiology, Ziv Medical Center, Israel \\ ${ }^{2}$ The Azrieli Faculty of Medicine, Bar-Ilan University, Israel
}

*Corresponding author: Saher Srour, MRI unit, Department of diagnostic radiology, Ziv Medical Center, Israel; The Azrieli Faculty of Medicine, Bar-Ilan University, Israel

\begin{tabular}{|c|}
\hline ARTICLE INFO \\
\hline Received: 㓞 April 19, 2021 \\
\hline Published: April 30, 2021 \\
\hline
\end{tabular}

Citation: Saher Srour, Rabih Fares. Role of Modern Sonography in the Assessment of Knee Pathologies: Review of a Large Series of Patients. Biomed J Sci \& Tech Res 35(3)2021. BJSTR. MS.ID.005707.

Keywords: Ultrasound; Knee; Meniscus; Sensitivity; Tear

\begin{abstract}
Introduction: Meniscal injuries of the knee are common. Acute meniscal tears occur most often from twisting injuries; chronic degenerative tears occur in older patients and can occur with minimal twisting or stress. Meniscal cysts present with symptoms of pain often associated with a palpable mass at or adjacent to the knee joint and are frequently accompanied by horizontal or complex meniscal tears. Bedside ultrasound (US) is being used more frequently to assess patients with acute knee injuries. Ultrasound allows the skilled practitioner to examine the knee dynamically and to compare injured and uninjured joints. Different previous studies showed inconsistent results regarding the sensitivity and specificity of US examination: some showed that sonography is not accurate enough to be used as the only imaging modality for diagnosing tears of the knee menisci, and another few studies showed a sensitivity of US matching that of MRI. In the daily practice we still get many US referrals with a question of meniscal tear.
\end{abstract}

Objectives: The main purpose of this study is to determine the role of sonography in detecting knee pathologies, and if it can be used as the main single exam for diagnosing meniscal injuries.

Materials and Methods: A retrospective review of US exam results done by musculoskeletal radiologist in Ziv Medical Center, for 358 patients/370 knees between the years 2013-2015. The prevalence of different knee pathologies shown on US exam will be calculated, and the relationship between these pathologies will be checked by statistical methods; Chi square and logistic regression, and the sensitivity and specificity of US will be calculated in relation to MRI, which is the Gold Standard non-invasive modality for detecting meniscal tears.

Importance: The results and conclusion of this study have an important economic value and effect. They will affect the daily practice of physicians to decide to which diagnostic examination they refer their patients and to help them decide how to act when getting the results of the US exam.

Results: The number of patients who were tested for knee pain using US was 358, where $203(56.7 \%)$ were females and $155(43.3 \%)$ were males. The mean age was 45.9 years. Out of the 358 who underwent knee US, 330 (92.2\%) had at least one pathology seen, in 28 (7.8\%) the scan was negative. 212 (59.2\%) had Baker's cyst, 14 (3.9\%) had parameniscal cyst, $53(14.8 \%)$ had ligament pathology, $165(46.1 \%)$ had tendon pathology, $101(28.2 \%)$ had osteoarthritic changes and in 140 (39.1\%) other pathologies were found. In $4(1.1 \%)$ was lateral sub-luxation, and 79 (22.1\%) had medial sub-luxation. Lateral meniscal tear in 17 (4.7\%) and a medial meniscal tear in $122(34.1 \%)$ of patients. Out of all patients who underwent US scan for their knees, 24 (7\%) had also knee MRI, in whom $6(25 \%)$ had lateral meniscal tear and $14(58.3 \%)$ had a medial meniscal tear. In $28(7.8 \%)$ the scan was negative. The Sensitivity of the US scan to detect medial tear was $78.57 \%$, and the Specificity was $70 \%$, PPV was $78.57 \%$ and NPV was $70 \%$. For a 
lateral tear, the Sensitivity was $50 \%$ and the Specificity was $94.4 \%$, PPV was $75 \%$ and NPV was $85 \%$. There was no major difference between genders who complaints of knee pain, $56.7 \%$ females and $43.3 \%$ males. The median age is 45.9 years; no prevalence of knee pain is seen in one age group. In $92.2 \%$ of the patients who were tested by US for a knee pain there was a pathology seen, so we can conclude that, with a skilled radiologist, US is a reliable first line modality for knee pain complaints. The most common pathology found in knee US was Baker's cyst in 59.2\% of patients. On the contrary, the least common pathology found was lateral sub-luxation in $1.1 \%$ of patients. When we compared the results of the US of knee with the MRI, we found a Sensitivity of $78.5 \%$ and a Specificity of $70 \%$, PPV of $78.57 \%$ and NPV of $70 \%$ for medial meniscal tears. For lateral meniscal tears the Sensitivity was $50 \%$ and the Specificity was $94.4 \%$, PPV was $75 \%$ and NPV was $85 \%$. These results are consistent with those published in the literature.

Conclusion: There is a high correlation between medial meniscal tear and osteoarthritic changes in the knee joint, likewise between lateral meniscal tear and lateral subluxation and parameniscal cyst. Therefore, US exam is a good first line modality in patients with knee complaints, and patients with positive results for meniscal tear, meniscal subluxation, degenerative medial changes or parameniscal cyst, should be directed toward MRI exam and further investigation and treatment. However, patients with negative US exam and high suspicion of internal knee derangements (meniscal tear, cruciate ligament tear) should be directed for MRI examination, due to NPV around 70$85 \%$.

\section{Introduction}

The knee is the largest joint in human body considering its intraarticular cartilage volume and area. Among the other body joints, it is mostly prone to traumatic injuries, wear and septic or inflammatory arthritis. The most common complaint is sensitivity at its medial side, and least common in lateral side. Meniscal tears are common injuries with many aetiologies, traumatic injuries in young patients and wear injuries in older patients are the most common ones. Para-meniscal cyst can present as a painful palpable mass inside or near the knee, and it's commonly a result of longitudinal or complex meniscal tear. Several studies have shown that the accuracy of physical examination in detecting meniscal tear depends on the type and location of the tear. The ultrasound modality is used in diagnosis of a painful knee. MRI can detect presence and type of meniscal tear, it is the most sensitive noninvasive method in detecting small tears. In one meta-analysis, MRI has sensitivity and specificity of $91.4 \%$ and $81.1 \%$ respectively in detecting medial tears, as for the lateral meniscus tear, sensitivity $76 \%$ and specificity of $93.3 \%$ [1-5].

Some of the advantages of MRI are non-invasive, high sensitivity and specificity, no ionizing radiation and is not operator dependent. Disadvantages include not readily available, expensive, has some contraindications and is time consuming. As a result, in 1989, US started to be used for detecting pathologies in painful knee. Its advantages are cheap, widely available, fast, dynamic examination, the ability to simultaneously compare both knees, safe and almost no contraindications. On the other hand, it is operator dependent, highly influenced by the experience of the performer and the resolution depends on the machine frequency. Studies to test the sensitivity and specificity of the US in detecting meniscal tears have shown different results:

a) Some showed that the US is not sensitive enough (sensitivity of $60 \%$ and specificity of $21 \%$ ), others showed sensitivity ranging between $83-86 \%$ and specificity of $69 \%$ in comparison to MRI scan which was used as the definitive diagnostic method.

b) Because of theses contradicting results, we decided to conduct this study to test the role of US in detecting different pathologies in the knee joint, such as: Baker's cyst, parameniscal cyst, meniscal extrusion, meniscal tears etc. and discover if there are any relations between those pathologies. The sensitivity and specificity of the US will be calculated in patients participating in the study who also underwent MRI or Arthroscopy of the knee [6-10].

\section{Study Assumption}

US is a good first line modality for knee pathology, it can detect a wide range of pathologies and sign for meniscal tears, although it is not sensitive nor specific enough for direct detection of meniscal tears, hence it can be used as a screening modality, and when it rises the suspicion of meniscal tear, it is recommended to proceed to MRI.

\section{Study Expectations}

Diagnosing a wide range of different pathologies in the knee joint using US. There are causative relations between different pathologies. US is a good screening test for a painful knee, but it is not sensitive enough in detecting meniscal tears. 


\section{Meanings and Implications}

The results of our study have practical clinical impact; they would help clinicians to forward their patients to the right examination. Also, they have a financial impact, which could save money and time by referring the patients to the right examination and avoiding unnecessary ones.

\section{Methods and Materials}

We conducted a retrospective analytical study. Results of the US examination of the knee done in Ziv Medical Centre by the same senior musculoskeletal radiologist were collected from the patient's medical files. The distribution of the pathological findings was calculated. Pathologies that were tested are - lateral meniscal tear (LMT), medial meniscal tear (MMT), medial and lateral subluxation, Baker's cyst, parameniscal cyst, degenerative changes, tendon pathology, ligament pathology and other pathologies. The relation between different pathologies and meniscal tears was also considered. Finally, we compared US results with MRI and arthroscopy results to determine the specificity, sensitivity, PPV and NPV of US in diagnosing meniscal tears [11-14].

\section{Devices}

Two US devices were used, PHILIPS IU 22 and PHILIPS EPIQ 5G, using musculoskeletal intended transducer 5-12 MHZ.

\section{Statistical Analysis}

CHI square test was used to determine the relations between different pathologies. Logistic regression test, while the dependent variable is meniscal tear, and the explanatory variables are the other pathologies. This regression was applied to all pathologies against all other pathologies. Statistical significance needed is $5 \%$ and test strength is $80 \%$. Sample size was all participants who underwent knee US by the same radiology musculoskeletal senior physician, in the radiology department in Ziv Medical Centre, this factor will eliminate the interindividual variability.

\section{Patient Criteria}

\section{Inclusion Criteria}

a) All patients who underwent ambulatory US examination of the knee

b) Both genders included

c) Age above 18 years

d) All examinations were held by the same senior musculoskeletal radiologist.

\section{Exclusion Criteria}

a) Patients who were referred to exclude space occupying lesion

b) Pregnant patients

c) Patients with impaired judgments (low mental status)

d) Children under the age of 17 years.

\section{Results}

Overall number of participants was 358, 203 females (56.7\%) and 155 males (43.3\%), mean age 45.9 years. Out of the 358 patients, in 212 (59.2\%) Baker's cyst was detected, 14 (3.9\%) parameniscal cyst, 53 (14.8\%) ligament pathology, 165 (46.1\%) tendon pathology, 101 (28.2\%) degenerative changes and 140 (39.1\%) other pathologies. In 4 patients (1.1\%) lateral subluxation was found versus 79 (22.1\%) with medial subluxation. Lateral meniscal tears were detected in $17(4.7 \%)$ and medial meniscal tear in 122 (34.1\%) - (Figure 1).

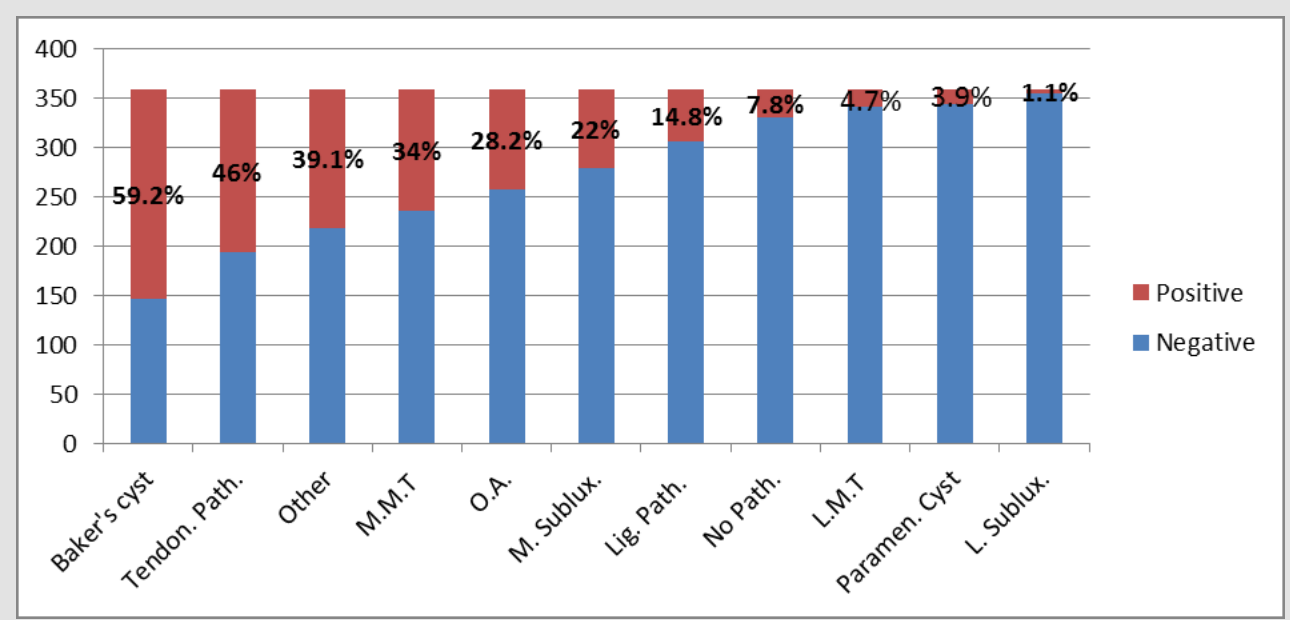

Figure 1: Incidence of different pathologies in knee US examination.

O.A: Osteoarthritis; M. Sublux: Medial Subluxation; L. Sublux: Lateral Subluxation; Lig. Path: Ligament Pathology; No Path: No Pathology; Paramen. Cyst: parameniscal cyst; LMT: Lateral Meniscal Tear; MMT: Medial Meniscal Tear. 
Out of all patients who underwent US, 24 (7.0\%) have done also MRI, 6 (25\%) had LMT and 14 (58.3\%) had MMT. In 28 (7.8\%) there was no pathology detected in US or MRI?. The sensitivity for MMT was $78.5 \%$ and specificity of $70.0 \%$, PPV 78.5\% and NPV of $70.0 \%$. For LMT sensitivity was $50.0 \%$ and specificity of $94.4 \%$, PPV $75.0 \%$ and NPV of $85.0 \%$. The calculations were made based on comparison with the MRI results, which considered the gold standard non-invasive test for detection of meniscal tears (Tables 1 \& 2). Arthroscopy was performed in 7 (2.5\%) patients: in 3 (42.9\%)

Table 1: Comparison between results of MRI and US for MMT. was LMT and in 5 (71.4\%) MMT. Because of these low numbers of patients, we couldn't calculate the sensitivity and specificity in comparison to arthroscopy. In those who passed MRI, we found high relations between: MMT seen in MRI and in US $(\mathrm{P}=0.016)$ and degenerative changes in US $(\mathrm{P}=0.032)$. LMT seen in MRI and in US $(\mathrm{P}=0.010)$ and lateral subluxation in US $(\mathrm{P}=0.009)$. Also, there was a trend toward relation between para-meniscal cyst and LMT $(\mathrm{P}=0.08)$ (Figures 2-12).

\begin{tabular}{|c|c|c|c|c|c|}
\hline & & & \multicolumn{2}{|c|}{ M.M.T MRI } & \multirow{2}{*}{ Total } \\
\hline & & & $\mathbf{0}$ & 1 & \\
\hline \multirow{6}{*}{ M.M.T US } & \multirow{3}{*}{0} & Count & 7 & 3 & 10 \\
\hline & & \% M.M.T US & $70 \%$ & $21.4 \%$ & $91.4 \%$ \\
\hline & & \% M.M.T MRI & $70 \%$ & $30 \%$ & $100 \%$ \\
\hline & \multirow{3}{*}{1} & Count & 3 & 11 & 14 \\
\hline & & \% M.M.T US & $30 \%$ & $78.57 \%$ & $100.0 \%$ \\
\hline & & \% M.M.T MRI & $21.4 \%$ & $78.57 \%$ & $16.7 \%$ \\
\hline \multirow{3}{*}{\multicolumn{2}{|c|}{ Total }} & Count & 10 & 14 & 24 \\
\hline & & \% M.M.T US & $100 \%$ & $100 \%$ & $100.0 \%$ \\
\hline & & \% M.M.T MRI & $41.7 \%$ & $58.3 \%$ & $100.0 \%$ \\
\hline
\end{tabular}

Table 2: Comparison of results between MRI and US for LMT.

\begin{tabular}{|c|c|c|c|c|c|}
\hline & & & \multicolumn{2}{|c|}{ M.M.T MRI } & \multirow{2}{*}{ Total } \\
\hline & & & $\mathbf{0}$ & 1 & \\
\hline \multirow{6}{*}{ M.M.T US } & \multirow{3}{*}{0} & Count & 17 & 3 & 20 \\
\hline & & \% L.M.T US & $85.0 \%$ & $15.0 \%$ & $100.0 \%$ \\
\hline & & \% L.M.T MRI & $94.4 \%$ & $50.0 \%$ & $83.3 \%$ \\
\hline & \multirow{3}{*}{1} & Count & 1 & 3 & 4 \\
\hline & & \% L.M.T US & $25.0 \%$ & $75.0 \%$ & $100.0 \%$ \\
\hline & & \% L.M.T MRI & $5.6 \%$ & $50.0 \%$ & $16.7 \%$ \\
\hline \multirow{3}{*}{\multicolumn{2}{|c|}{ Total }} & Count & 18 & 6 & 24 \\
\hline & & \% L.M.T US & $75 \%$ & $25 \%$ & $100.0 \%$ \\
\hline & & \% L.M.T MRI & $100.0 \%$ & $100.0 \%$ & $100.0 \%$ \\
\hline
\end{tabular}

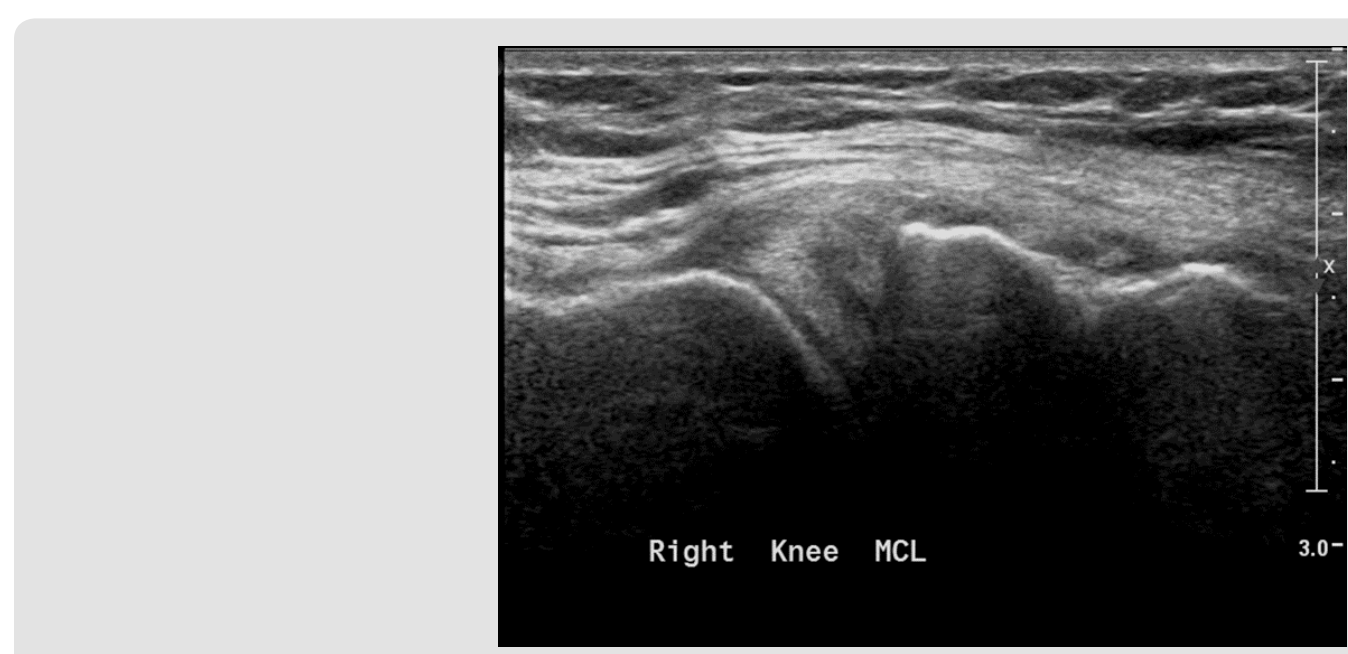

Figure 2: MMT in US (thick arrow). 


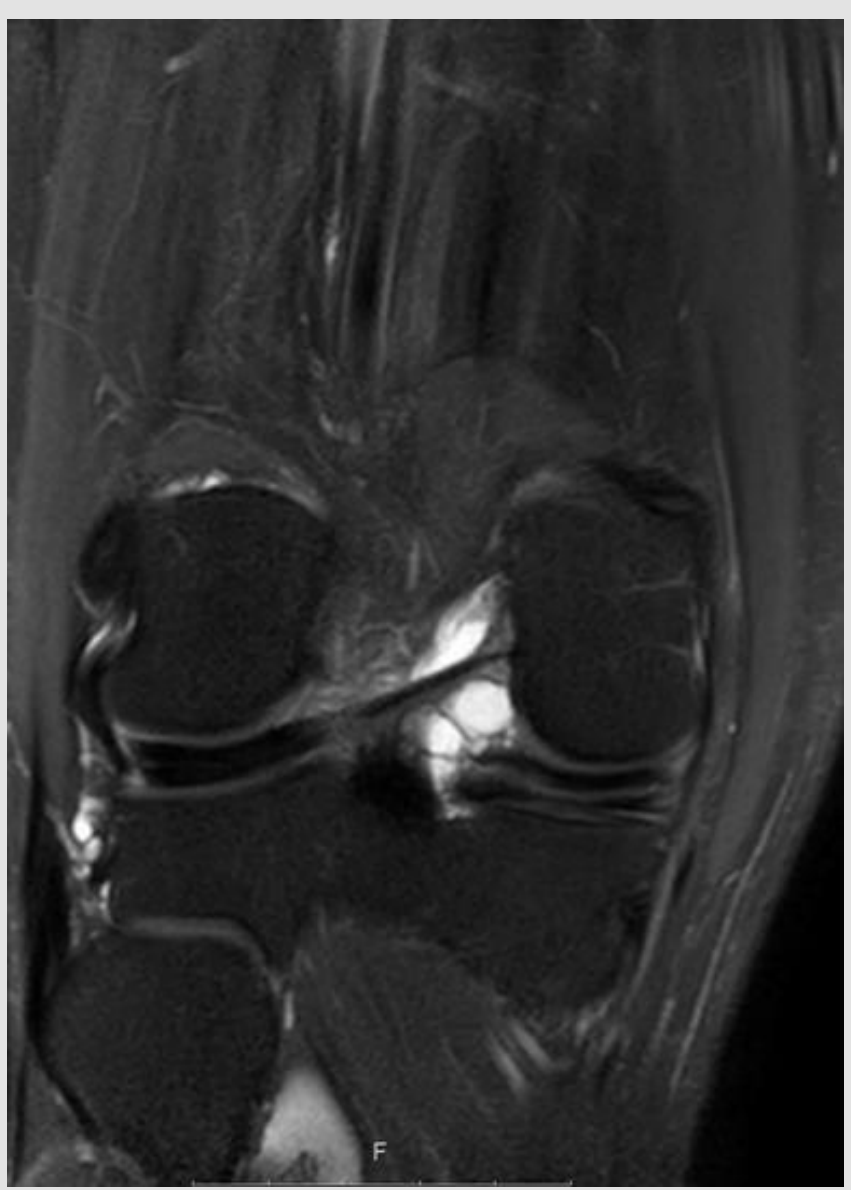

Figure 3: MMT (thick arrow) and parameniscal cyst (thin arrow) in MRI.

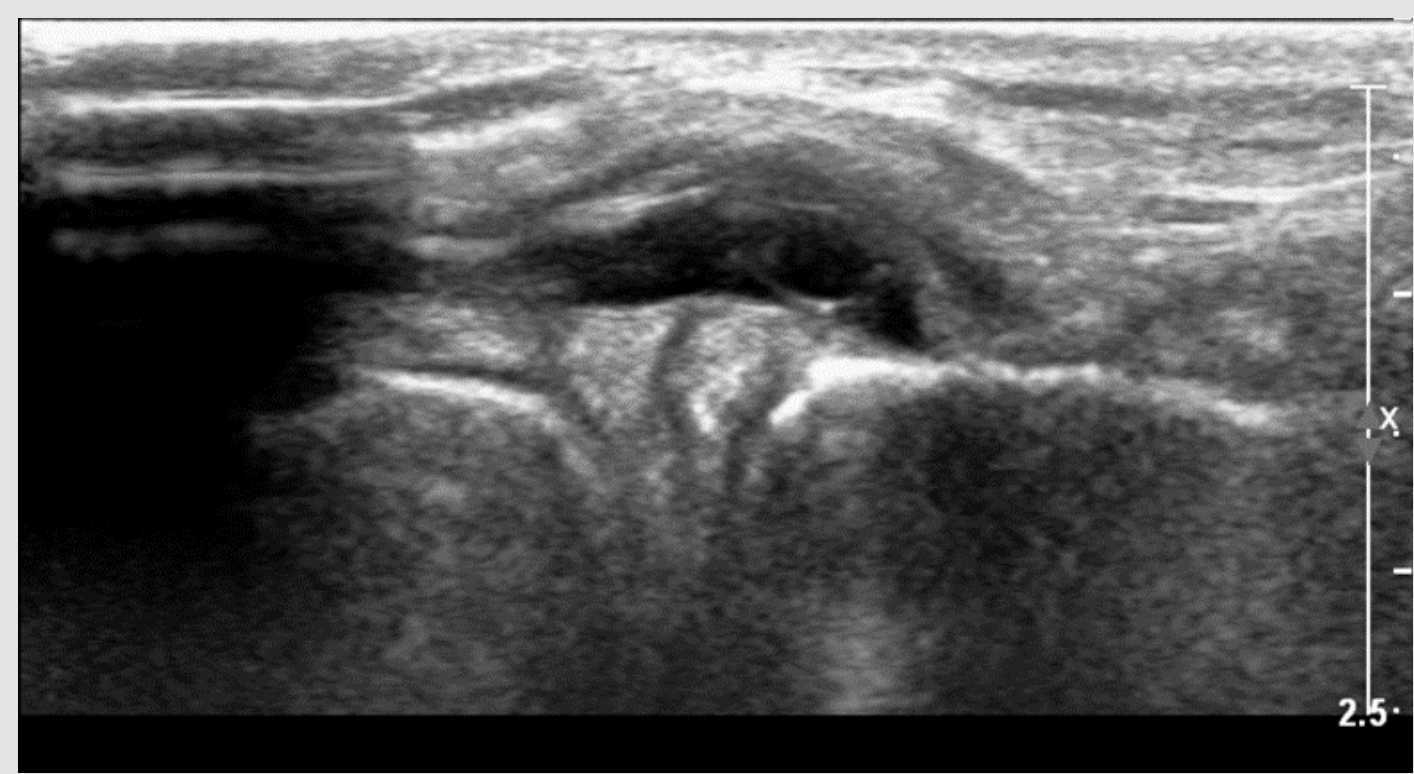

Figure 4: LMT (thick arrow) and parameniscal cyst (thin arrow) as seen in US. 


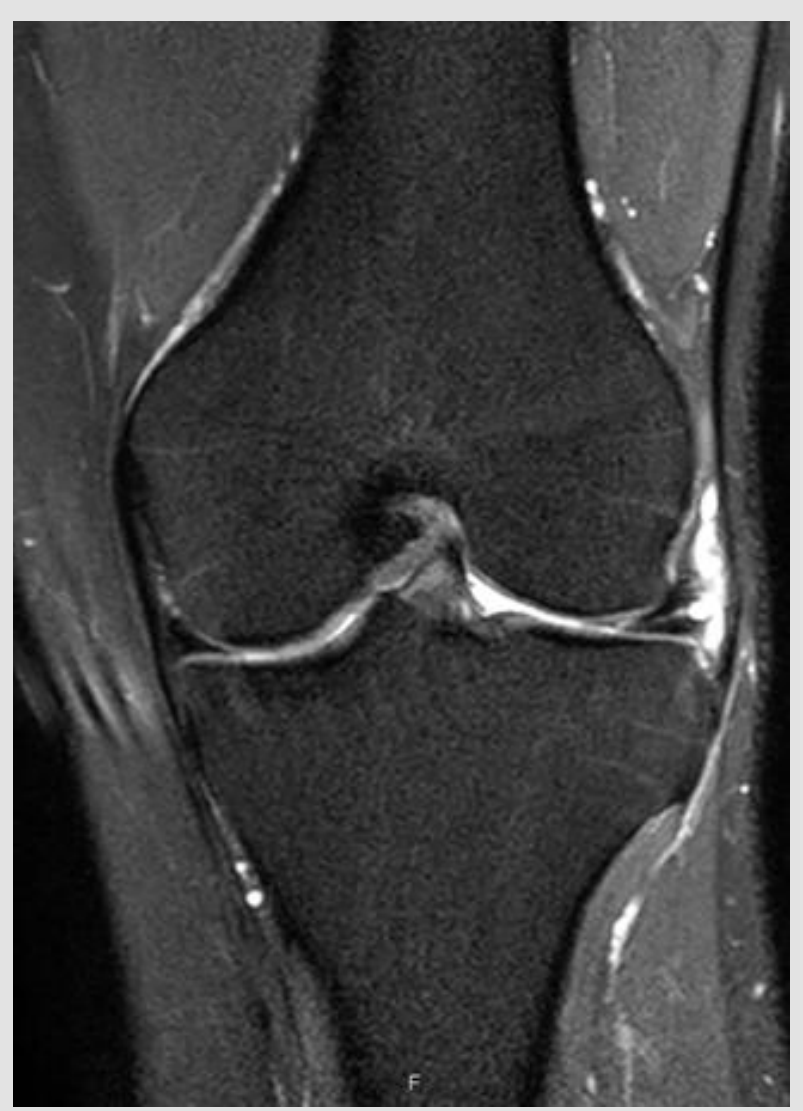

Figure 4: LMT (thick arrow) and parameniscal cyst (thin arrow) as seen in MRI (same patient as in Figure 4).

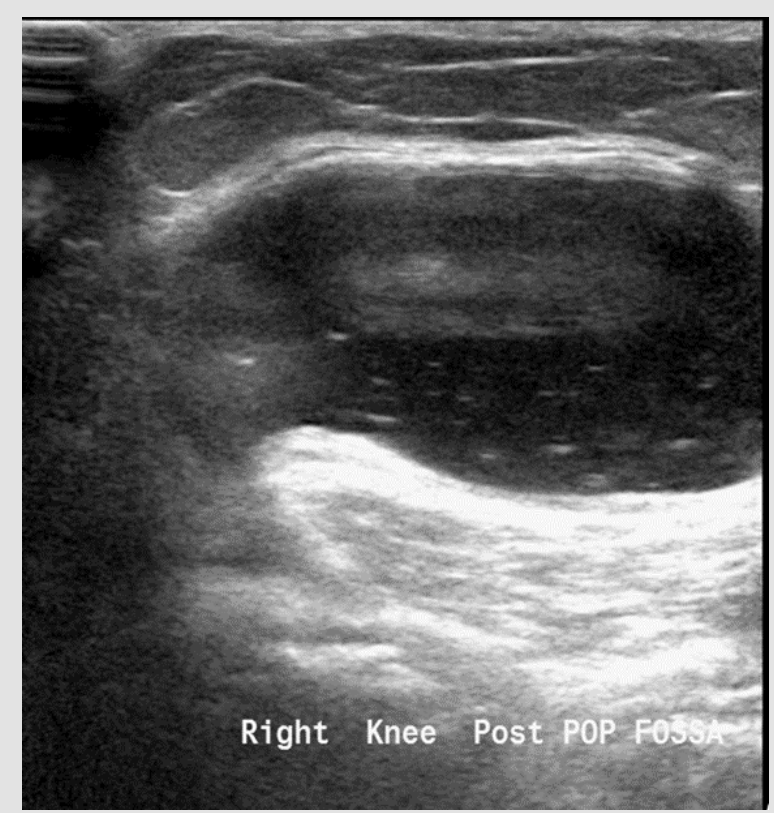

Representative examples of different knee pathologies as seen in US:

Figure 6: Baker's cyst - located in the popliteal fossa between the tendon of the semimembranosus and the medial gastrocnemius. It's size ranges from few millimeters to few centimeters, and it is a common reason for knee complaints, it can cause pain, or it might be asymptomatic. It also can be in relation to intraarticular effusion from mechanical or inflammatory aetiologies. 


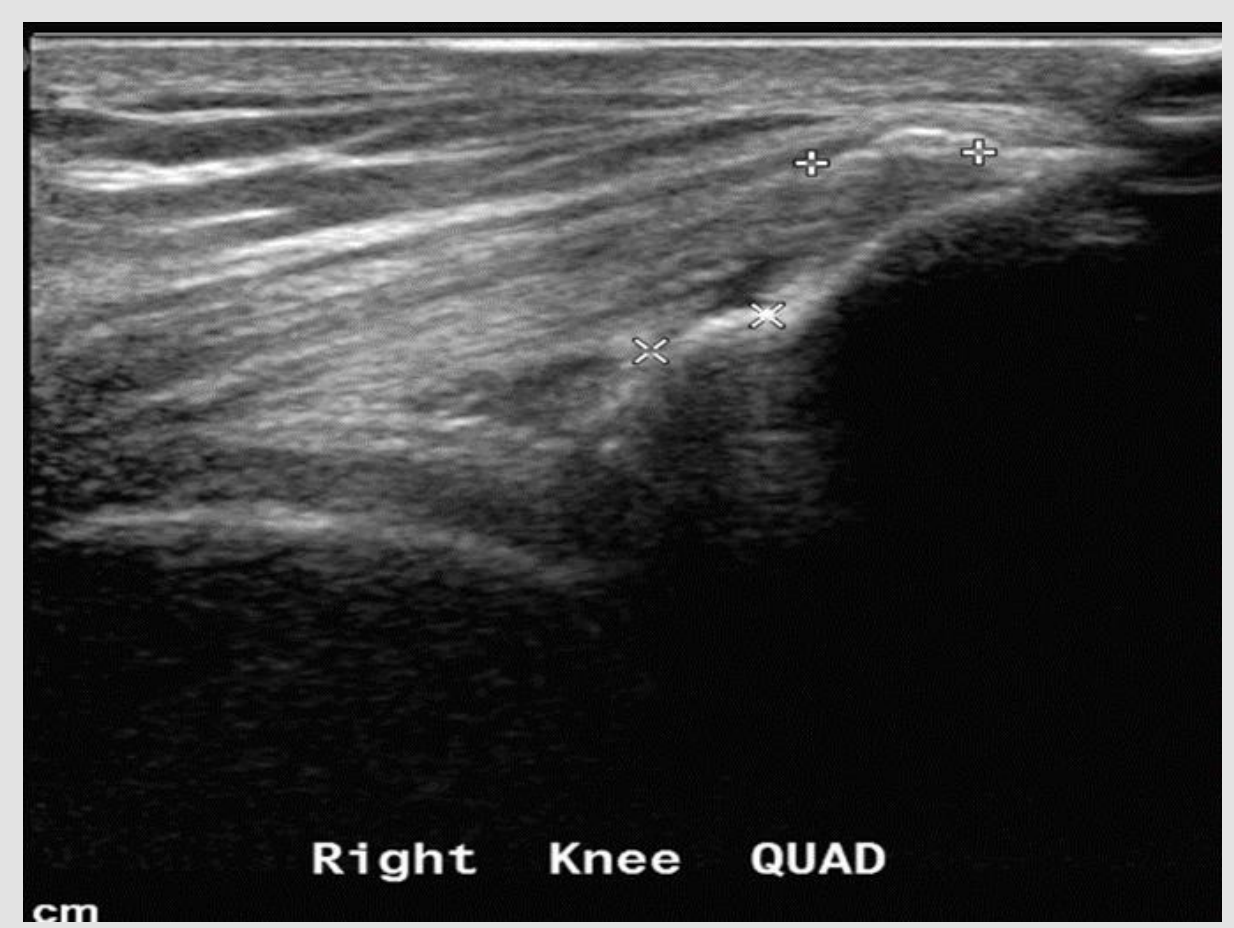

Figure 7: Quadriceps insertion tendinopathy - the quadriceps tendon appears hypoechoic with calcifications at its insertion into the patella.

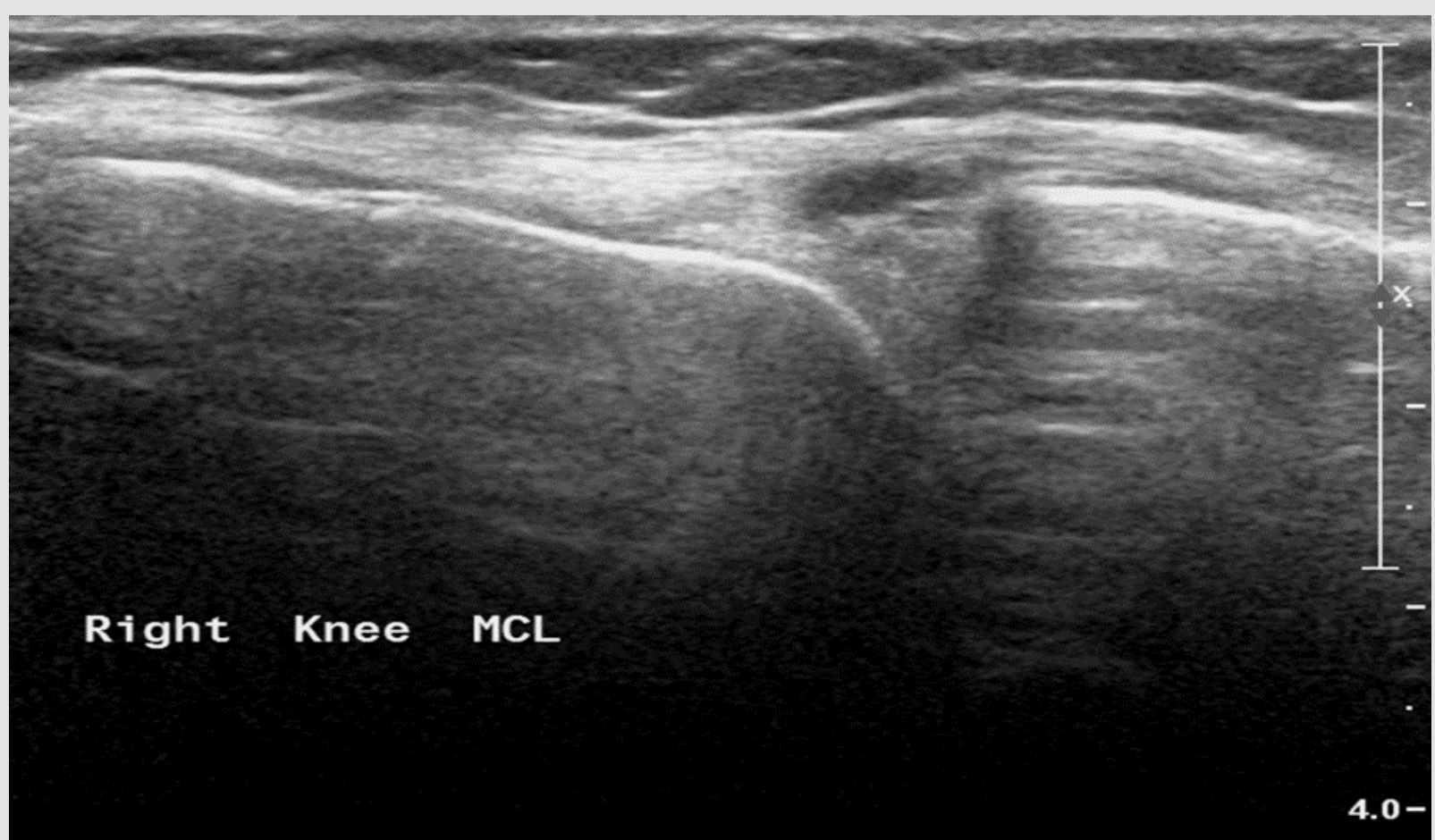

Figure 8: Parameniscal cyst - anechoic structure, sharply demarcated, adjacent to the medial meniscus. 


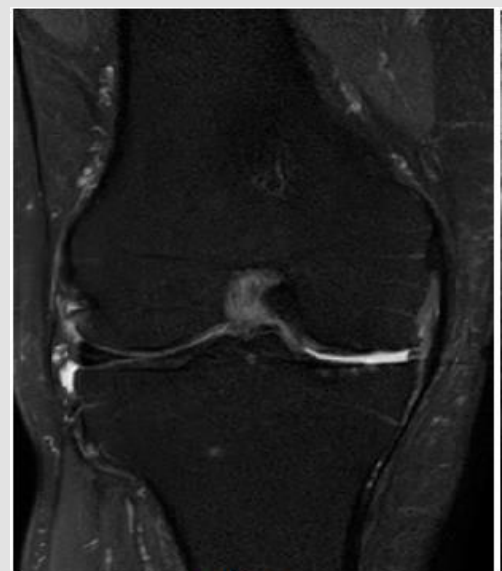

Figure 9

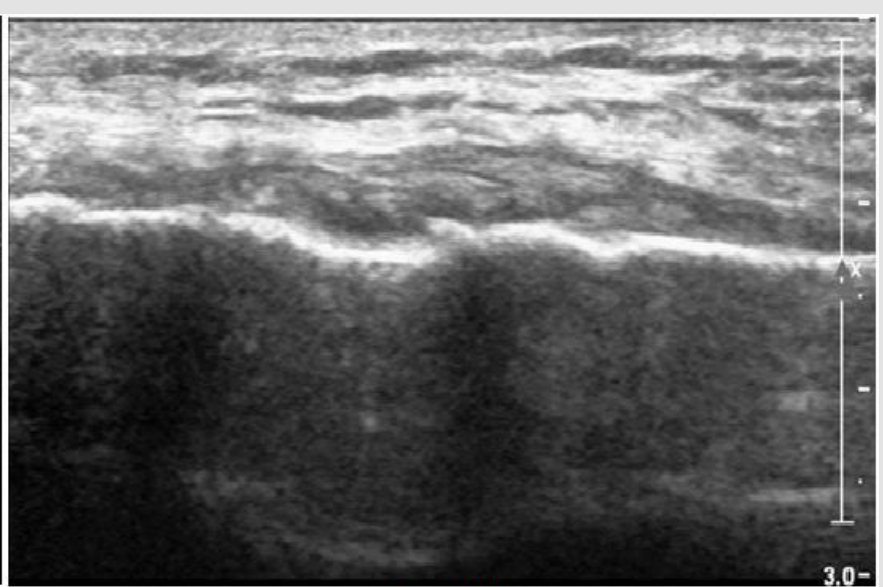

Figure 10

Figure 9 \& 10: Osteoarthritis presented in MRI and US - severe narrowing of the medial compartment, disappearance of the medial meniscus, cartilage erosion, and mild subchondral oedema from both sides the medial compartment.

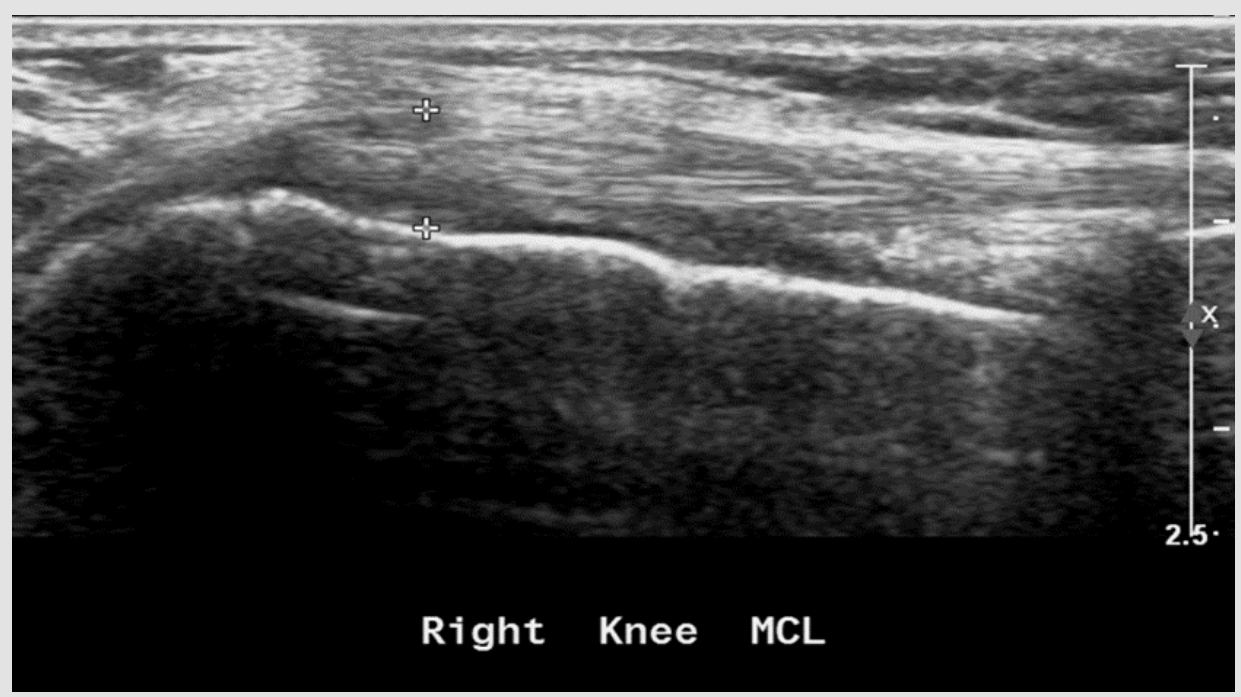

Figure 11: Medial collateral ligament partial tear - thickening, hypoechoic proximal part of the ligament with fluid around it.

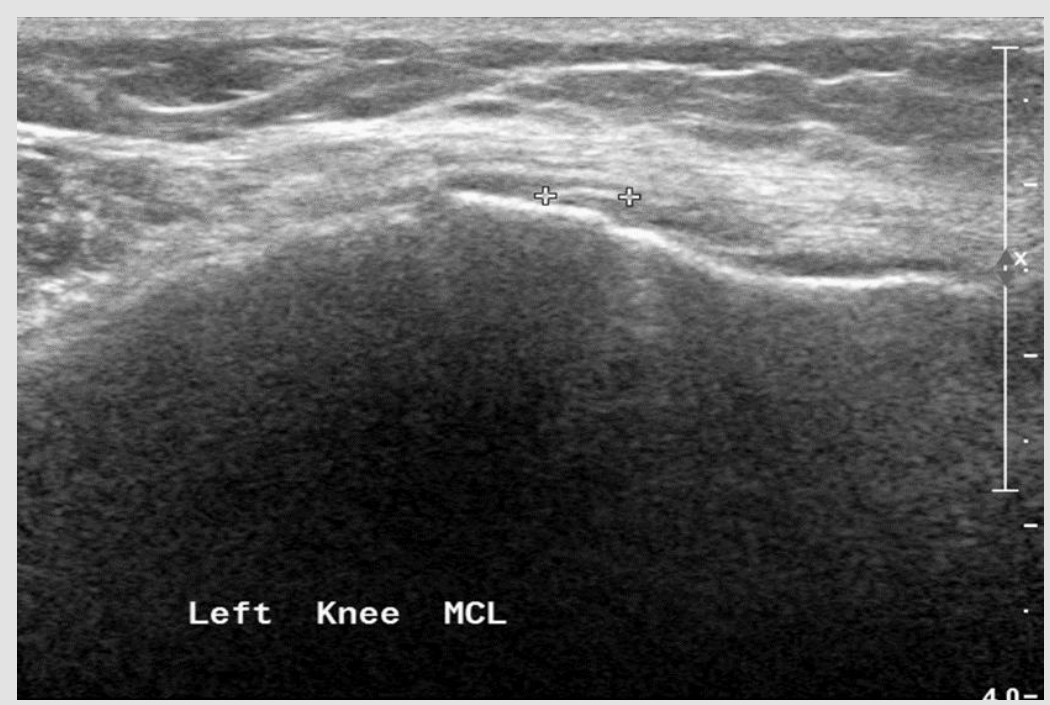

Figure 12: Pellegrini Stieda disease - thickening with calcifications at the insertion of the ligament to the Femur, a manifestation of chronic changes because of old partial tear. 


\section{Discussion}

Knee pain is a common complaint, more pronounced at the medial compartment; as a result, many patients are referred to diagnose the aetiology. Nowadays, US exam is considered a first line modality for patients with knee pain. The ability of the US in revealing the above-mentioned pathologies is manifested by that $92.2 \%$ of the referred patients in our study were diagnosed with at least one knee pathology. Reasonable explanations for the inability to find pathology in US examination are referred pain to the knee area or pathologies that cannot be diagnosed with US. In our study, the most common finding was Baker's cyst with incidence of 59.2\% (212 patients), higher than showed in previous studies (6.6 - 25\%). The least common pathology was lateral meniscal subluxation $(1.0 \%)$. There was no gender difference in the referred patients, $56.7 \%$ females and $43.7 \%$ males. The median age is 45.9 years which shows that there is no predilection for a certain age group

The lateral and medial menisci are important structures in the knee joint. They function as shock absorbers and transfer the weight from the axial skeleton to the calves. Meniscal tear may result from degenerative changes or from acute trauma [15-17].

Risk factors for meniscal tears include age above 60 years, males, discoid meniscus, occupational factors such as professions that require recumbency, squatting and stair climbing. Meniscal tear mostly results in knee pain and functional deterioration. Tear configuration are longitudinal, horizontal, radial, complex and sometimes with a teared flap. In some cases, arthroscopy is needed to eliminate the pain and to improve the functional status of the patients. The characteristic finding in US for meniscal tear is hypoechoic line. In one study, sensitivity, specificity, PPV and NPV for US as compared to arthroscopy were $85.4 \%, 85.7 \%, 67.3 \%$ and $94.4 \%$ respectively. The statistical parameters did not differ for medial and lateral meniscal tears. Age, gender, BMI, body weight, physical activity, injury mechanism and time passed from injury were not statistically significant factors in determining the efficacy of US. The highest sensitivity (more than 90\%) was derived from MMT in patients under BMI 25. The highest specificity (above 90\%) was in patients with LMT injured in a in rotatory mechanism during a physical activity also in new injuries (less than one month since the injury). The PPV was above $90 \%$ in recent injuries only (less than a month), on the other hand, NPV less than $90 \%$ in males with sport injury with LMT [18-20].

In our study, MMT was seen in 34.1\% (122) of patients while only $4.7 \%$ (17) were with LMT. When we compared the results of US against MRI (which is the non-invasive gold standard modality), we found sensitivity of 78.5\% and specificity of 70.0\%, PPV 78.5\% and NPV 70.0\% for MMT. As for LMT 50.0\%, 94.4\%, 75.0\% and $85.0 \%$ respectively. In patients which underwent also MRI, we found significant concordance between the results of the MRI and US in diagnosis of MMT $(\mathrm{P}=0.016)$ and LMT $(\mathrm{P}=0.01)$. Also, a significant relation between lateral subluxation seen in US and LMT seen in MRI ( $\mathrm{P}=0.009)$, between degenerative changes in US and MMT in MRI $(\mathrm{P}=0.032)$, and trend toward relation between para-meniscal cyst and LMT $(\mathrm{P}=0.08)$. The above results highlight the importance of the US, performed by a skillful radiologist, in detecting meniscal tears as compared to MRI. Also, in young patients with degenerative changes in the medial compartment seen using US, meniscal subluxation and parameniscal cyst, it is reasonable to refer the patient to MRI for further evaluation of meniscal tear as a possible reason.

\section{Conclusion}

US examination is cheap, widely available, fast, dynamic, performed in real time and lack ionizing radiation.

In skillful hands, it can detect a wide variety of knee pathologies in patients referred with knee pain. We have found sensitivity, specificity, NPV and PPV for detection of meniscal tears similar to published results in the professional literature, as compared to MRI. US examination can be used as a first modality in suspected meniscal tears. Patients with detected meniscal tears, parameniscal cyst or medial degenerative changes shall be further referred to MRI because of the high probability of meniscal tears. Although, if the US examination is negative but the clinical signs and symptoms are highly suggestive of meniscal tears or other knee joint pathology, like ACL $\backslash$ PCL tears, it is recommended to resume evaluation by MRI because of NPV of $70.0 \%-85.0 \%$ in US.

\section{References}

1. Anderson BC (1999) Office Orthopedics for Primary Care: Diagnosis and Treatment. $2^{\text {nd }}(E d n)$, WB Saunders, Philadelphia, Chapter 11: 213-215.

2. Binfield PM, Maffulli N, King JB (1993) Patterns of meniscal tears associated with anterior cruciate ligament lesions in athletes. Injury 24(8): 557-561.

3. Rutten MJ, Collins JM, van Kampen A, Jager GJ (1998) Meniscal cysts: Detection with High-Resolution Sonography. AJR 171(2): 491-496.

4. Scholten RJ, Devillé WL, Opstelten W, Bijl D, van der Plas CG, et al. (2001) The accuracy of physical diagnostic tests for assessing meniscal lesions of the knee: a Meta-Analysis. J Fam Pract 50(11): 938-944.

5. Konan S, Rayan F, Haddad FS (2009) Do physical diagnostic tests accurately detect meniscal tears?. Knee Surg Sports Traumatol Arthrosc 17(7): 806-811.

6. Dzoleva Tolevska R, Poposka A, Samardziski M, Georgieva D (2013) Comparative analysis of diagnostic methods in meniscal lesions. Prilozi 34(3): 79-84.

7. Yan R, Wang H, Yang Z, Ji ZH, Guo YM (2011) Predicted probability of meniscus tears: comparing history and physical examination with MRI. Swiss Med Wkly 141: w13314.

8. Lee SY, Jee WH, Kim JM (2008) Radial tear of the medial meniscal root: reliability and accuracy of MRI for diagnosis. AJR Am J Roentgenol 191(1): 81-85.

9. Gray SD, Kaplan PA, Dussault RG (1997) Imaging of the knee. Current status. Orthop Clin North Am 28(4): 643-658.

10. Bureau NJ, Kaplan PA, Dussault RG (1995) MRI of the knee: a simplified approach. Curr Probl Diagn Radiol 24(1): 1-49. 
11. Crawford R, Walley G, Bridgman S, Maffulli N (2007) Magnetic resonance imaging versus arthroscopy in the diagnosis of knee pathology, concentrating on meniscal lesions and ACL tears: a systematic review. Br Med Bull 84: 5-23.

12. Boos N, Bugyi J (1989) The Value of Meniscus Sonography of the Knee Joint. Unfallchirurg 92(9): 435-439.

13. Casser HR, Fustin M (1993) Current development in Ultrasonography of the Meniscus. Orthopadie 22(5): 307-316.

14. Azzoni R, Cabitza P (2002) Is There a Role for Sonography in the Diagnosis of Tears of the Knee Menisci?. J of Clinical Ultrasound 30(8): 472-476.

15. AA Shetty, AJ Tindall, KD James, J Relwani, KW Fernando (2008) Accuracy of Hand-Held Ultrasound Scanning in Detecting Meniscal Tears. The J of Bone \& Joint Surgery 90(8): 1045-1048.

\section{ISSN: 2574-1241}

DOI: 10.26717/BJSTR.2021.35.005707

Saher Srour. Biomed J Sci \& Tech Res

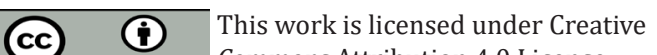

Submission Link: https://biomedres.us/submit-manuscript.php
16. Picerno V, Filippou G, Bertoldi I, Adinolfi A, Di Sabatino V, et al. (2014) Prevalence of Baker's cyst in patients with knee pain: An ultrasonographic study. Reumatismo 65(6): 264-270.

17. Iagnocco A, Meenagh G, Riente L, Filippucci E, Sedie AD, et al. (2010) Ultrasound imaging for the rheumatologist XXIX. Sonographic assessment of the knee in patients with osteoarthritis. Clin Exp Rheumatol 28(5): 643-646.

18. Maffulli N, Longo UG, Campi S, Denaro V (2010) Meniscal tears. Open Access J Sports Med 1: 45-54.

19. Snoeker BA, Bakker EW, Kegel CA, Lucas C (2013) Risk factors for meniscal tears: A systematic review including meta-analysis. J Orthop Sports Phys Ther 43(6): 352-367.

20. Wareluk P, Szopinski KT (2012) Value of modern sonography in the assessment of meniscal lesions. Eur J Radiol 81(9): 2366-2369.

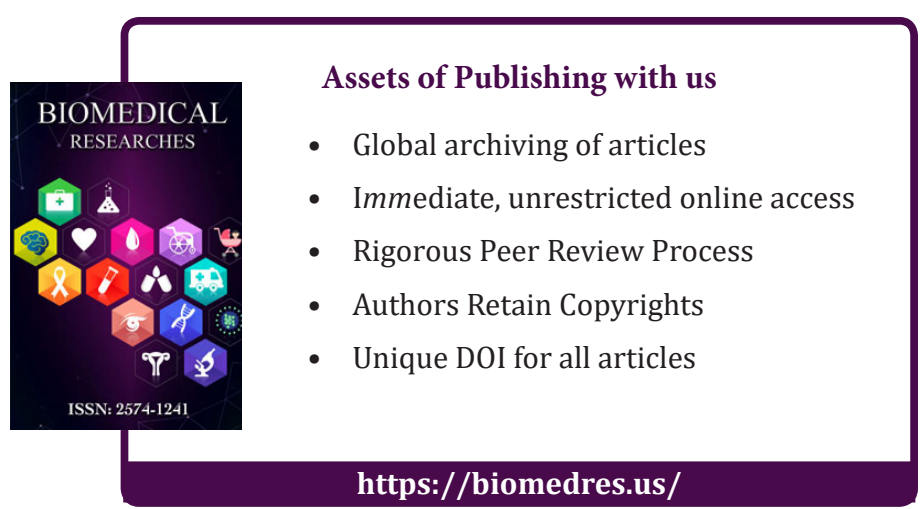

